Témoigner Témoigner. Entre histoire et mémoire

Getuigen Revue pluridisciplinaire de la Fondation Auschwitz

118 | 2014

Au nom des victimes. Dictature et terreur d'État en Argentine, Chili et Uruguay

\title{
Agenda en français et en anglais
}

Agenda in French and English

Agenda in het Frans et het Engels

\section{(2) OpenEdition}

\section{Journals}

Édition électronique

URL : http://journals.openedition.org/temoigner/1222

DOI : 10.4000/temoigner. 1222

ISSN : 2506-6390

Éditeur :

Éditions du Centre d'études et de documentation Mémoire d'Auschwitz, Éditions Kimé

Édition imprimée

Date de publication : 1 octobre 2014

Pagination : 8-13

ISBN : 978-2-84174-674-3

ISSN : 2031-4183

Référence électronique

"Agenda en français et en anglais », Témoigner. Entre histoire et mémoire [En ligne], 118| 2014, mis en ligne le 01 octobre 2015, consulté le 23 octobre 2020. URL : http://journals.openedition.org/ temoigner/1222 ; DOI : https://doi.org/10.4000/temoigner.1222 

d'Oradour-sur-Glane.

Exuger.

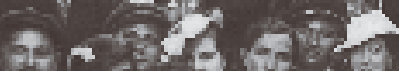

a $y=1,4$ man

ars

EXPOSITION

\section{Hommage aux victimes}

d'Oradour-sur-Glane

À l'occasion des $70^{\mathrm{e}}$ commémorations du 10 juin 1944, le Centre de la mémoire, en partenaria avec lAssociation Nationale des Fur-Gles des Martyrs doradoursur-Glane, rend hommage aux

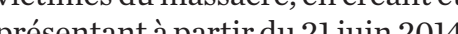
presentant a partir du 21 juin 2014 Visposition temporaire «Oradour, les portraits individuels des victimes et leur vie quotidienne vicdes photrap de famille, des photographies de famille, de tiosse ou de groupe, cette exposition permet de donner un visage un chiffre, etde dévoiler rervies a des from des fragments de vie au travers des instants figès par ces clichés. Cette centrée sur les victimes, átél'oc- casion de réaliser une grande collecte, qui a permis de récolter des photographies jusquálors inconaprès, la démarche destretricedes après, la dénarchés nazis n'avait pas réussi a élininer

$\diamond \grave{A}$ partir du 20 juin 2014

$\diamond$ Centre de la mémoire d'Oradour Lauze, 87520 Oradour-sur-Glane.

le livre de jeunesse de l'universite Goethe de Francfort (Hans(IFH lirHA, sur la littérature de jeu-

$\checkmark$ 10-12 septembre 2014.

$\rightarrow$ Goethe-Universität -

rrankfurtSenckenberganlage

3160325 Frankfurt am Main.

EXPOSITION

COLLOQUE

\section{4 / 2014 - First World War. War Childhood and Youth, Literature,}

\section{Commemorative Culture}

Colloque international organisé par l'Institut de recherche sur

\section{«Paris libéré, Paris} photographié, Paris exposé » Dans le cadre des commémorations célébrant la Libération de Paris, le musee Carnavalet organise Potographé, Pars libéné, Paris

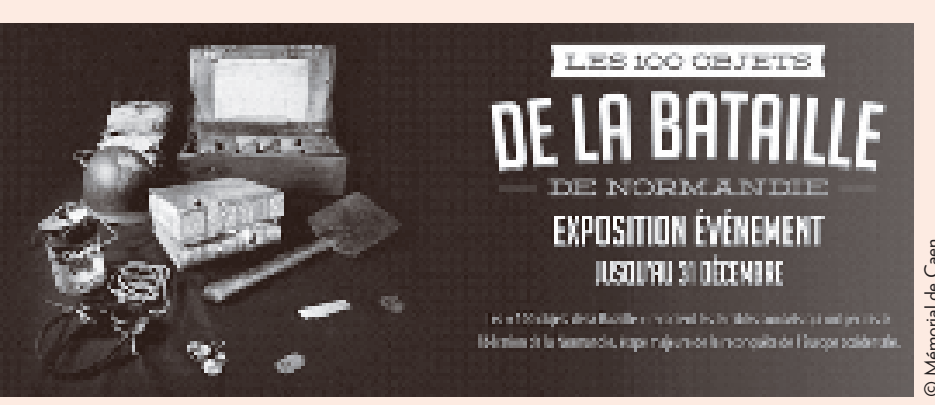

EXPOSITION

\section{"LES 100 OBJETS DES 100 JOURS}

DE LA BATAILLE DE NORMANDIE »

Pour porter un regard différent sur cet épisode majeur de la Libération, le Mémorial a choisi de présenter 100 objets, civils et militaires, témoins des émouvant... chaque objet a une histoire à raconter.

$\diamond$ Du 31 mai au 31 décembre 2014 $\triangle$ Mémorial de Caen - Esplanade Général Eisenhower, 14000 Caen.
www.memorial-caen.fr

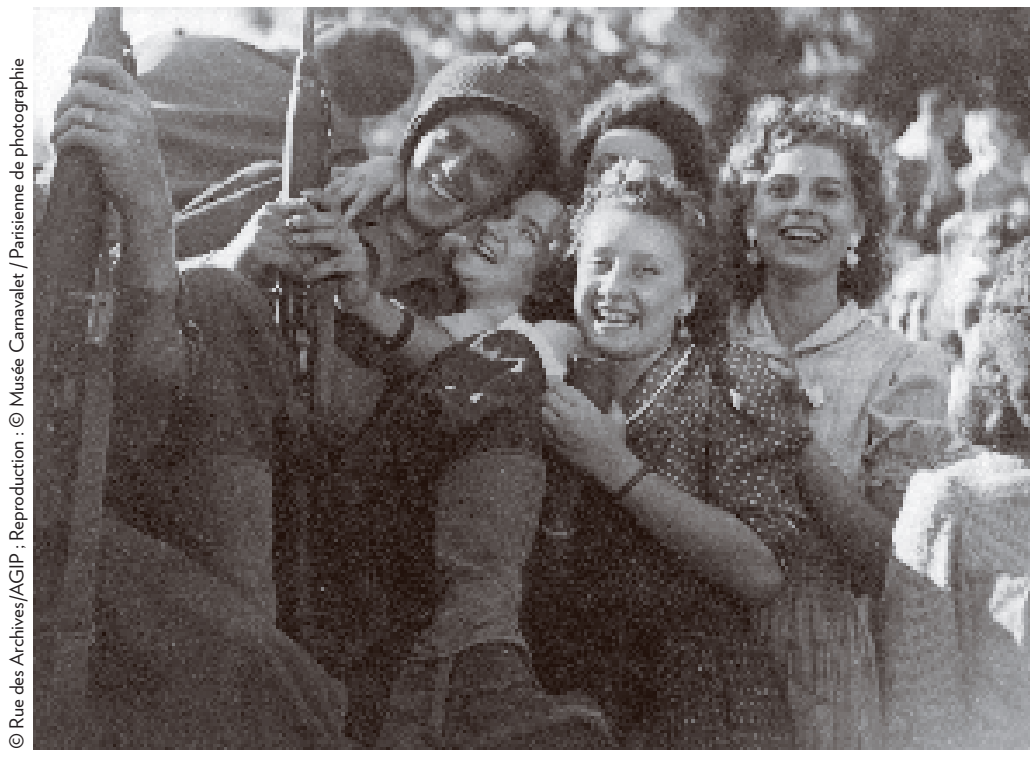

$\uparrow$

décrire l'histoire de la Libération de Paris grâce à des films et des photographies. Il sagit également historique musée Carnavalet ambition d'apporter un regad neuf sur d'apporter rexpo concept dexposition historique contre où se mêlent différ rengénérations. Le mé fait aptè leneriations. Le nusce fait appel à lartiste Jochen Gerz pour renoucerémedive

$\diamond$ Du 11 juin 2014 au 8 février 2015.

$\Rightarrow$ Musée Carnavalet-Histoire de 75003 Paris. www.carnavalet.paris.fr
7 .

EXHIBITION

\section{4-1918.}

The First World War

Taking fourteen salient places as points of departure the exhibition
L'accueil fait aux soldats » - 25 août 194

offers a survey of events and thei different contexts. Using individual biographies, it reveals the very wevent were experisnced and helps visicors to understand how dranataffected the people.

$\diamond 29$ May to 30 November 2014

$\diamond$ Deutsches Historisches Museum - Unter den

MUSÉE

Le Musée de la mémoire de Belleau 1914-1918

Au cœur du village de Belleau, à $00 \mathrm{~km}$ de Paris, ce Musée perpéMarnes qui ont conbattu au Bois .

Le Bois de Belleau est le lieu d'un triste et néanmoins célebrebataille tue le souvenir et la Mémoire des germanique aux troupes alliées ont une majorité composée d un lieu unique pour. Cest deven un Mar Dans un pre-Uir espace musé Dans un premis espace du est présentée surle cimetière ricain et le Corps des Marines. Un secondespacepropose desexposi-

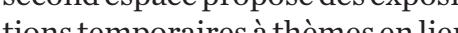
avecta Prom

$\Leftrightarrow$ Le musée est ouvert du

Smai au 11 novembre.

$\diamond$ Place du Général-Pershing

02400 Belleau. www.musee-memoire-

MUSÉ

14-18 - Bruxelles

l'heure allemande

Comprenez grâce à l'exempl ruxellois, la façon dont la guerre Xxe sicte-

Dombreux documents (cartes postales, photos, breuses caricatures, faites nor des artistes bruxellois de l'épor des qui cirulaient sous le manteau. partir du 20 août 1914 et 50 mo partint 20 aruts durvivre sous occuption. On l'oublie trop souvent, mais Bruxelles est une des seules cais Bruxelles co rope, et la plus grande ville du cous le joug allemand. $\diamond$ Du 21 août 2014 au 3 mai 2015. $\triangle$ Musée de la Ville de Bruxelles Grand Place -1000 Bruxelles. www.14-18.bruxelles.be 
EXPOSITION

\section{C'est le débarquement !}

À l'occasion du $70^{\mathrm{e}}$ anniversaire de la libération du territoire, le Centre d'Histoire de la Résistance et de la Déportation consacre une exposition temporaire aux débarquements de Normandie et de Provence. L'exposition propose une vence. L'exposition propose une innersion dans ces evenements découres dojets à travers disich es parcours et points de viant es parcours et points de vue de phéêtre desóérations

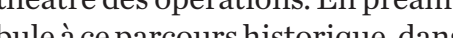
政 le cadre dun partenariat avec Lyon Des travaux des auteurs de bande lessinée

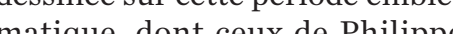
t ceux de Philipp

$\diamond$ Du 12 juin 2014 au 4 janvier 2015 . $\diamond$ Centre d'Histoire de la Résistance et de la Déportation - Espace Berthelot - 14, avenue Berthelot,
69007 Lyon. www.chrd.lyon.fr

OUVERTURE

\section{Bastogne War Museum}

Le Bastogne War Museum contitue un nouveau lieu de mémoire diale en algique. Il vourre mondiale en Belgique. Il vous propose et interative des causes, événethe prismedera prismede Le Bastogne War Museum gravite diale, depuis ses racines jusqua l'automne 1944, ainsi quautour de

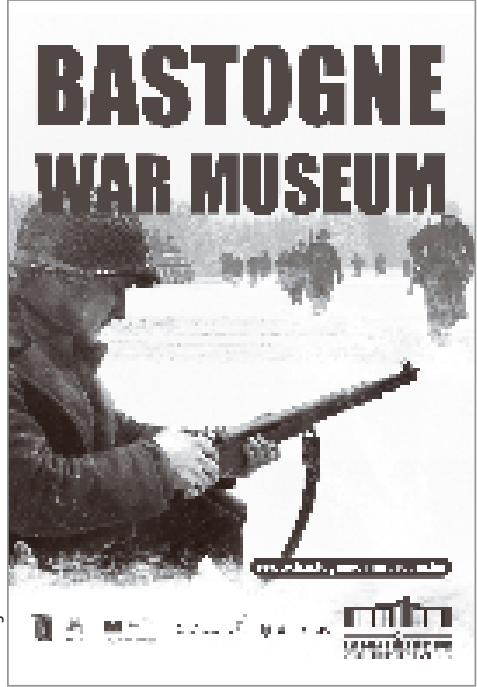

$\uparrow$

la bataille des Ardennes. Les principaux faits de guerre et la souffrance des soldats, afin de mieux comprendre la Grande Histoire, vous y sont présentés. Le centre qui pouvait bos aperçu de ce à quils porantlocention la de batrille ou time bén deénémoire originale de ce centre e nouveau pascours nón. Le nouveau parcours muséal est

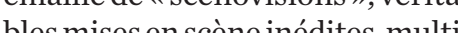
besmisesensconeinédites, nult$\rightarrow$ Colline du Mardasson, 5 6600 Bastogne.

Co

EXHIBITION

\section{Truth and Memory: British} Art of the First World War

Truth and Memory will be the largest exhibition and first major War art for al Brish 100 ye Wors will include work by some of Britain's most important artists of the drawn mainly from. Using collection this exhibition collection, this exhibition will assess the innediste First World War.

$\diamond 19$ July 2014 to 8 March 2015.

$\diamond$ Imperial War Museum London
- Lambeth Road -London, Greater London - England, SE1 6HZ. www.iwm.org.uk

EXHIBITION

\section{The First World War}

\section{in Jewish Memory}

Caught in conflict between belonging and exclusion, the First World War provides a central reference point for German-Jewish commemorative culture. Of particuthe works of Jewish artists the Herms of Jewish artists such as Hernann struck, Jacob Steinhardt and Ernst Oppler, who were soldiers at the front. Most of the objects on display in the cabinet everyday life of war

$\diamond 3$ July to 16 November 2014

$\diamond$ Jewish Museum Berlin -

Lindenstrafe 9-14 - 10969 Berlin.

www.jmberlin.de

EXPOSITION

\section{Rwanda 1994 :}

\section{Rwánocide des Tutsi}

À travers une disposition scénographique reproduisant les mémoriaux rwandais et visant à approcher levènement par une

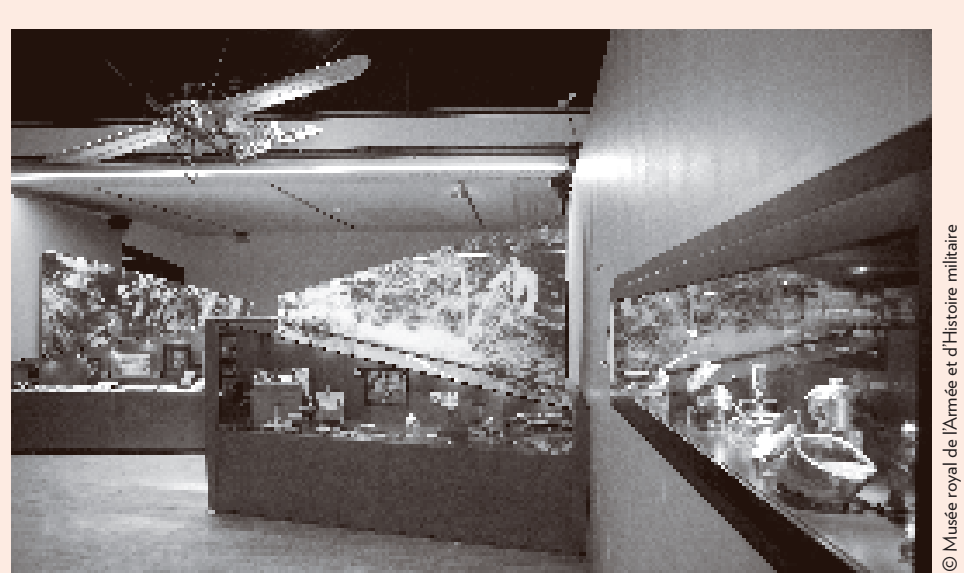

EXPOSITION

\section{4-18, C'EST NOTRE HISTOIRE !}

Au travers d'objets authentiques, de décors, de témoignages, d'installation conflit a détermin en Belgique occupée constituent une part importante de l'exposition. Vous redécouvrirez également Thistoire de la mobilisation des Etats européens qui ont cherche à secourir la Belgique. Enfin, un parcours spécial propose un portrait croisé du Roi Albert $1^{\text {er }}$ et de l'Empereur Guillaume II.

$\Leftrightarrow$ Du 26 février 2014 au 26 avril 2015.

$\rightarrow$ Musée royal de l'Armée et d'Histoire militaire

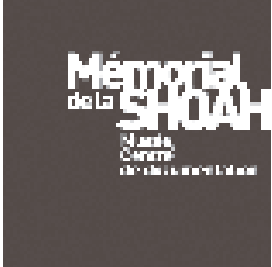

archives sonores, vidéo ou écrites, l'exposition permet de restituer la ssé toujours vivan uu Rwanda, vingt ans après.

$\diamond$ Du 11 avril au 5 octobre 2014 $\triangle$ Mémorial de la Shoah - 17, rue
Geoffroy-l'Asnier, 75004 Paris. www.memorialdelashoah.org/rwanda/
EXPOSITION

\section{Regards sur les ghettos}

Près de 500 photographies peu ces des ghettos sontexposés ement, ces lieux d'exactions ont encenten de clich́s. Que nous dontant de clichès. Que nous donnent à fonction? I'exp Quition te le $\diamond$ Jusqu'au 28 septembre 2014

$\diamond$ Mémorial de la Shoah.

regards-ghettos.memorialdelashoah.org
EXPOSITION

Trop humain. Artistes des $20^{\mathrm{e}}$ et $21^{\mathrm{e}}$ siècles devant la souffrance

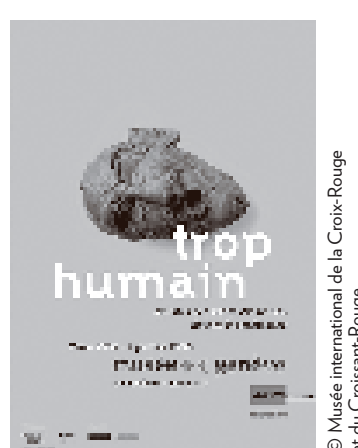

Le musée montre un choix d'œuvres qui envisagent la souffrance infligée par des hommes à 'autres hommes. Un ensemble de pièces est réuni allant des image historiques d'Otto Dix, consacrée áétion création plus récente illustree, $\mathrm{par}$ exemple, par les corps douloureux de Loulse Bourgeois (19), enpasde Pablo Picasso et de Zoran Mušič $\diamond$ Jusqu'au 4 janvier 2015

$\diamond$ Musée international de la CroixRouge et du Croissant-Rouge Avenue de la Paix 17 - 1202 Genève.

EXPOSITION

2014 Horchposten -

L’installation sonore « 2014 Horchposten - Postes d'écoute 1914 est un espace de mémoire de la Premiere Guerre mondiale que le viduellement. Son focus se porte 
sur le front entre la France et l'Allemagne, limage delennemi constitele fil deproposer au visiteur unchangeent l'époque. Ainsi, l'ins lentioni de eteque. Ainsi, linstallation perGrande Guerre : un soutive de

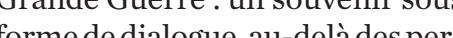
forme de dialog : and dela desperceptions nationales. L'installation tituts Goethe en France.

$\Leftrightarrow$ Du per Octobre au 11 Novembre
2014 au Goethe-Institut de Paris.

$\diamond$ Goethe-Institut de Paris

17, avenue d'léna, 75016 Paris.

EXPOSITION

\section{Visages et vestiges}

Présentée à la gare de l'Est à Paris, lieu emblématique du départ des hotogration photographique de Didier Pazery ente 1996 et 2007 qui mettente entre 1996 et 2007 qui mettent en conflit alors qu'ils étaient cents conflit alors quils étaient cente-

$\diamond$ Du 23 juin au 20 novembre 2014.

$\diamond$ Gare de l'Est, 75010 Paris.

www.expo14.com

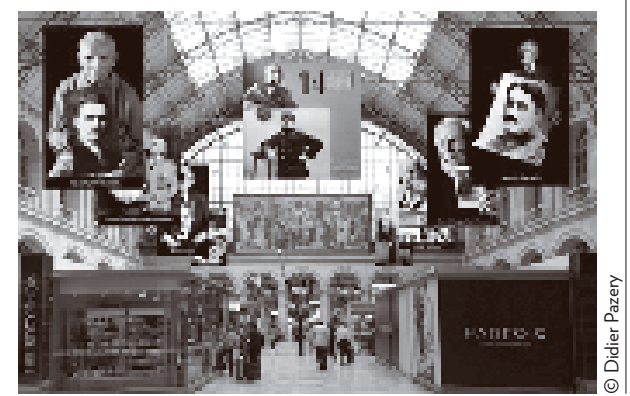

EXPOSITION

\section{Les désastres de la guerre} 1800-2014

ette exposition explore la Cencé du $19^{e}$ siècle jusqù̀ nos jours. Elle du siecle jusquá nos jours. Ell de dése ansi du mouvenent guerres. L'exposition s'intéress à gure ving Lexpostion sinteresse à une vingtaine de confits, avec des cocus particullers sur les guenres napoleoniennes, les Première et Seconde Guerres nond

$\diamond$ Du 28 mai au 6 octobre 2014

$\Leftrightarrow$ Musée du Louvre-Lens www.louvrelens.fr

EXPOSITION

\section{Loin de la querre? Les} émigrants belges en

\section{Prérique pendant la}

Le musée Red Star Line racont 'histoire des Américains d'oriHhistoire des Americains d'origine belge qui, pendant les années èrene, étaient coupés de leur dere patrie et deleur fanille. Comment sest passéelaguerrepour eux? Et quelrôle Line pendant ce temps-là?

$\diamond$ Du 26 septembre 2014 au 5 avril

$\triangle$ Musée Red Star Line -

Montevideostraat $3-2000$ Anvers.
www.redstarline.be

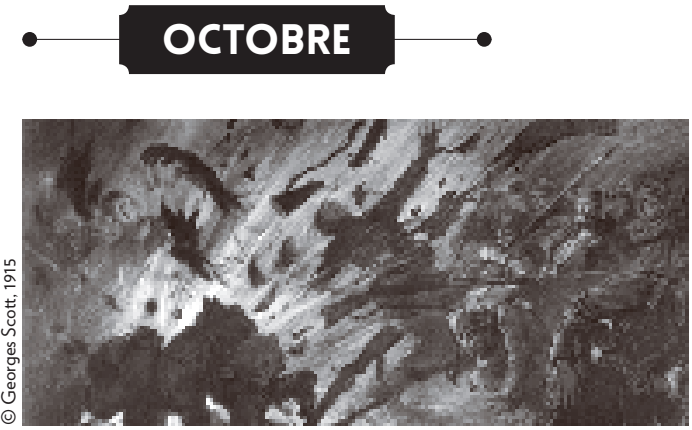

EXPOSITION

\section{Vu du front, représente}

la Grande Guerre

L'exposition s'intéressera à la manière dont les contemporains ont perçu et représenté le front. Comment les combattants ont-ils montré et pourquoi? Ilen ont-ils confroter le conflit ré sogia de lence inoüe du hamp de lenceinouí duchanpdebataille à laguerre héroïque telle quelle étai trés et le cinéma de l'époque

$\diamond$ Du 5 octobre 2014 au 25 janvi

2015

$\diamond$ Musée de l'Armée - Hôtel National des Invalides - 129, rue de Grenelle,

Grenell

ÉVÉNEMENT

\section{La Liste de noms/, \\ Front de Lumière'14}

Le 17 octobre 2014, GoneWest programme de commémoration de la province de Flandre-Occidentale, organisera un grand evénement participatif intitule Fron front tele quelle se pre ligne de front telle quelle se présentait en de fin 1914 après linondation de fin octobre. En outre, la liste
des 600000 victimes tombées sur

le territoire belge sera également projetée sur le monument Albert a Dixmue

$\diamond 17$ octobre 2014. www.gonewest.be

ÉVÉNEMENT

\section{Le pont flottant comme un} pont de la paix sur l'Escaut

L'un des pointsforts du programme de la ville d'Anvers autour du centenaire de la Première Guerre mondiale sera la reconstitution de 1914 ent de entre Le pont offre une occasion unique te visits et pied sur l'Escaut, dans le sillage de

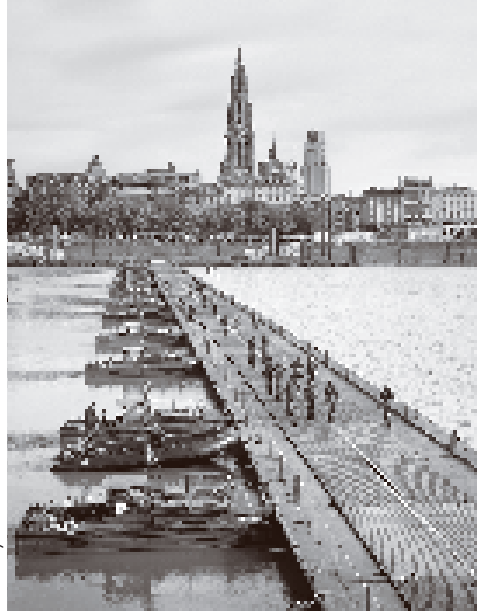

l'armée belge, mais aussi des plus 100000 réfugiés qui laissèrent derrière eux, il y a 100 ans, une ville bombardée.

$\diamond$ 3-5 octobre 2014

$\diamond$ Steenplein - 2000 Antwerpen.
EXPOSITION

\section{4-18 LA GUERRE EN IMAGES /}

\section{BRUGES EN GUERRE}

\section{La ville commémore la Grande Guerr}

à travers plusieurs expositions. Deux

expositions de photographie montrent

des photos de la Première Guerre

mondiale, sélectionnées par Carl

De Keyzer et David Van Reybrouck,

provenant de la collection de l'agence

Magnum. Sophie De Schaepdrijur

(cf. notre entretien dans ce numéro),

(1)

ville occupée, depuis la mobilisation en juillet 1914 jusqu'aux commémoration d'après-guerre en 1919-1920.

$\diamond$ Du 14 octobre 2014 au 22 mai 2015

$\diamond$ Stadshallen (Halles de la ville) - Markt 7 - 8000 Bruges.
EXPOSITION

Guerre à la guerre : es expressionnistes mands, 1914-1918

C'est l'histoire de la douloureuse désillusion née de la Grand Guerre que racontera l'expositio Guint de vue d'artistes allemands point de vue d'artistes allemands de leurs homologues français. $\Leftrightarrow$ Du 22 octobre 2014 au 22 décembre 2016

$\diamond$ Palais des Beaux-Arts -

Palais des Beaux-Arts -
Place de la République, 59000 Lille.

\section{NOVEMBRE}

COLLOQUE

\section{Entrer en guerre}

L’Université Paris Diderot-Paris 7, en partenariat avec le Centre interde la Grande Guerre de Péroria de la Grande Guerre de Péronne plinaire portant sur la question plinaire portant sur la question culier psychique social, esthétique psychice, social, esthetique que Guerre mondiale a provièré. Guerre mondiale a provoqué. guerre a-t-il été vécu, représenté, conś ?

$\diamond$ Du 20 au 22 novembre 2014.

$\triangle$ Université Paris 7 Denis Diderot -
5, rue Thomas-Mann, 75013 Paris. 